\title{
A nationwide study of adolescent psychosocial well-being one year after the outbreak of the COVID-19 pandemic
}

\author{
Tilmann von Soest ( $\boldsymbol{\nabla}$ t.v.soest@psykologi.uio.no) \\ University of Oslo \\ Michal Kozák \\ Department of Psychology https://orcid.org/0000-0002-5799-9922 \\ Ruben Rodriguez-Cano \\ University of Oslo \\ Dirkje Fluit \\ University of Oslo \\ Laura Cortés-García \\ University of Oslo \\ Vidar Ulseth \\ University of Oslo \\ E. F. Haghish \\ University of Oslo \\ Anders Bakken \\ Oslo Metropolitan University
}

\section{Article}

Keywords: COVID-19, human behavior, psychosocial consequences

Posted Date: May 25th, 2021

DOI: https://doi.org/10.21203/rs.3.rs-526074/v1

License: (c) (i) This work is licensed under a Creative Commons Attribution 4.0 International License. Read Full License

Version of Record: A version of this preprint was published at Nature Human Behaviour on January 20th, 2022. See the published version at https://doi.org/10.1038/s41562-021-01255-w. 


\section{Abstract}

]The COVID-19 pandemic has dramatically restricted adolescents' lives. We used nationwide Norwegian survey data collected from 2014-2021 ( $N=227,258$; ages 13-18) to address three essential questions: What are the psychosocial consequences of the pandemic? Are disadvantaged adolescents disproportionally affected by the pandemic? Do pandemic effects vary with geographical variations in infection rates and restrictions? Multilevel models revealed higher depressive symptoms and lower optimism during the pandemic than before the pandemic, even when accounting for the measures' time trends. Further, alcohol and cannabis use decreased, and screen time increased. Social relationships and conduct problems remained stable. Girls, younger adolescents, and adolescents from low socioeconomic backgrounds were disproportionally affected by the pandemic. The pandemic's effects varied little with municipality infection rates and restrictions. These findings can inform means and interventions to reduce negative pandemic effects and identify groups that need particular attention during and after the pandemic.

\section{Introduction}

\section{]}

On March 11, 2020, the World Health Organization declared the COVID-19 outbreak a pandemic. Since then, drastic measures to prevent the spread of the pandemic have been implemented worldwide. Imposed restrictions, such as school closures, physical distancing, and restrictions on recreational activities raise concerns about adolescents' wellbeing ${ }^{1,2}$. Understanding how the pandemic has affected psychosocial aspects in key arenas of adolescents' lives is a fundamental step towards supporting adolescents during and after the pandemic. Moreover, it is crucial to obtain knowledge of whether disadvantaged groups suffer disproportionally from the consequences of the pandemic. Here, to address these issues we use nationwide, large-scale, repeated cross-sectional data from more than 200,000 adolescents in Norway that were assessed comprehensively on key psychosocial aspects before the COVID-19 outbreak and one year into the pandemic. We examine: (1) potential psychosocial consequences of the pandemic, (2) whether disadvantaged groups are particularly vulnerable to the effects of the pandemic, and (3) whether the effects of the pandemic vary with geographical variations in infection rates and restrictions.

Despite the growing interest in studying the psychosocial consequences of the COVID-19 pandemic on the general population, our knowledge about its impact on adolescents is still limited. First, there is a lack of studies providing comprehensive and comparable assessments of a variety of psychosocial factors before and during the pandemic. Second, some recent longitudinal studies that compared adolescents' responses before and during the pandemic did not disentangle the effects of COVID-19 from normative development during the teenage years ${ }^{3,4}$. Repeated crosssectional studies comparing same-aged adolescents before and after the pandemic can account for developmental trends during adolescence. Third, most studies so far have been based on small-sized or convenience samples ${ }^{4,5}$, whereas there is a lack of large-scale, population-based studies that include information about geographical variations in infection rates and restriction measures and their association to adolescent psychosocial well-being. Fourth, although some studies examined adolescents' psychosocial well-being during the initial months of the pandemic ${ }^{4,6,7}$, the long-term effects are unknown.

Using nationwide Norwegian data from adolescents assessed with the same procedure in 157 municipalities before and one year into the pandemic, we provide knowledge about the psychosocial consequences of the pandemic in key arenas of adolescents' lives. We disentangle the sudden consequences of the pandemic from long-term time trends using multilevel societal growth curves ${ }^{8}$ and additionally examine the role of geographical variations in infection rates and restrictions. This study utilizes the by-far largest sample of teenagers to examine psychosocial consequences of 
the pandemic up to now. The results provide information that will better inform intervention and prevention measures to reduce the adverse psychosocial effects of the pandemic among adolescents.

\section{Previous research on the impact of the pandemic on adolescents}

In this article, we aim to examine how the pandemic has affected adolescents' lives in five key life domains. These life domains include social relationships, mental health, conduct problems and substance use, physical activity and screen time, and future life expectations. Concerning social relationships, we know that adolescent social life has substantively changed during the pandemic, due to infection control measures such as school closures and physical distancing ${ }^{9}$. Consequently, peer relationships may be disrupted in a time of social deprivation such as the present. In fact, one of the greatest concerns related to the pandemic reported by adolescents is not feeling connected to their friends ${ }^{4}$. In addition to peer relationships, the pandemic may also have affected relationships within the family: Financial insecurity, caregiving burden, and confinement-related stress may adversely affect parent-child relationships ${ }^{10}$. Indeed, in the early stage of the pandemic, many parents reported experiencing increased levels of distress ${ }^{11}$. Such stress, in turn, has been shown to increase the practice of harsher parenting styles, resulting in more conflicts and worsening relationships between parents and their children ${ }^{12}$. However, it has also been posited that changes in family routines and the increased amount of time and attention spent within the family may have had positive effects for some children and adolescents ${ }^{13}$. This study will examine how peer relationships and parental relationships have changed from before to during the pandemic.

In the domain of mental health, it has been suggested that the pandemic leads to increasing mental health problems in children and adolescents because of the unique combination of social isolation, economic recession, and disruptions in mental health care services for adolescents ${ }^{1,2}$. Moreover, large-scale studies in several countries have indicated that early in the pandemic, mental health problems increased among adults ${ }^{14-18}$. However, research on adolescents has not been conclusive. For example, data from 12 longitudinal studies primarily conducted in North America found that adolescent depressive symptoms increased significantly from before to during the first six months of the pandemic, whereas symptoms of anxiety remained stable ${ }^{3}$. However, these longitudinal studies do not disentangle effects of the pandemic from normative aging processes in adolescence. To illustrate, a Norwegian longitudinal study indicated that increases in depression and anxiety during the pandemic were caused by adolescents getting older, rather than by effects of the pandemic ${ }^{6}$. Large-scale repeated cross-sectional studies are therefore important to provide more information about the effect of the pandemic on adolescent mental health.

The effect of the pandemic on substance use and conduct problems is another field where research is scarce. The pandemic limits situations where adolescents consume alcohol and other drugs, such as unsupervised gatherings and parties with peers ${ }^{19}$. Research with five longitudinal adult samples in the UK showed that alcohol use decreased during the pandemic, and the largest reduction was found in the youngest cohort, born in $2001^{20}$. In contrast, a study in Canada assessing drug consumption in adolescents retrospectively indicated increased alcohol and cannabis use 5 . Regarding conduct problems, crimes committed by young people in the United States seemed to decrease during the pandemic ${ }^{21}$. Here, we follow this line of research by examining changes of substance use and conduct problems from before to one year into the pandemic.

There have been concerns that restrictions during the pandemic may lead to a decrease in physical activity for children and adolescents, which, in turn may have long-term negative health consequences ${ }^{22}$. A multi-wave survey in China seems to confirm these concerns by finding reduced physical activity in children and adolescents due to the pandemic ${ }^{23}$. However, a longitudinal study on children and teens aged 4-17 in Germany found that even though sports activity declined in the beginning of the pandemic, habitual physical activities increased, thereby leading to an overall 
increase in physical activity ${ }^{7}$. Both studies also found that recreational screen time increased ${ }^{7,23}$. Consequently, while taking time trends into account, in this study we examine the effects of the pandemic on physical activity and screen time among Norwegian adolescents.

Finally, the pandemic may have an impact on adolescents' expectations concerning their future life opportunities. The pandemic may have challenged adolescents' basic beliefs about living in a controllable world and about their physical safety and economic security. Moreover, the economic recession triggered by the COVID-19 pandemic is expected to hit those who are in the initial phase of their labour market career the hardest, such as young people ${ }^{24}$. Thus, adolescents might be more pessimistic about their future prospects. To our knowledge, the pandemic's impact on adolescents' future expectations has not been examined up to now; we expect it to influence adolescents' expectations negatively.

\section{Are marginalized groups disproportionately affected by the pandemic?}

Poverty and low education are consistently associated with social ills, lifetime problems, and higher morbidity and mortality, both among adults ${ }^{25,26}$ and adolescents ${ }^{27}$. Social disparities typically increase in times of crisis ${ }^{28}$, and European and US research have documented that the less educated and the poor are economically more severely affected by the COVID-19 pandemic ${ }^{29,30}$. However, less is known about whether the pandemic disproportionately affects disadvantaged adolescents in a variety of life domains and thereby widens social disparities. According to the literature, adolescents from a low socioeconomic background may be disproportionately affected by the pandemic, with a higher risk of living in crowded households, increasing family stress, and adverse economic and health effects of the pandemic ${ }^{11,31}$.

The adverse effects of the pandemic may also vary with other sociodemographic factors. For example, adolescent girls show more symptoms of anxiety and depression and ruminative coping styles than boys ${ }^{32}$ and may be affected more by the pandemic psychosocially. Two longitudinal studies have provided first evidence that the pandemic has led to increasing gender disparities in mental health problems in adolescents ${ }^{4}$ and adults ${ }^{14}$. However, to our knowledge, studies examining whether girls are more severely affected by the pandemic in psychosocial domains other than mental health are lacking. Finally, pandemic effects may differ according to age. Research on adults has shown that mental health problems have disproportionally increased in young adults (18-24 olds) ${ }^{14}$, but no studies have been conducted as yet on age differences in adolescents. Over the course of adolescence, multiple significant developmental changes occur progressively that correlate with the age and timing of pubertal maturation. These developmental changes may affect the ways in which individuals understand and respond to significant life stressors ${ }^{33}$. The effect of the pandemic may therefore differ according to age during adolescence; however, the nature of such potential differences is unknown.

\section{Do changes from before to during the pandemic vary with regional differences in infection rates and restriction measures?}

COVID-19 infection rates and the extent of the restrictions imposed vary considerably both across countries and across smaller geographical units within a country, such as municipalities ${ }^{34}$. It would therefore be important to examine to what degree higher infection rates and stricter restrictions affect adolescents' psychosocial well-being. A study on adults has found that people who predominately physically distanced themselves report higher levels of mental health problems ${ }^{17}$, thereby indicating that mental health problems may be higher in areas with more extensive distancing measures. However, there is a lack of studies that directly examine the association between infection rates, restriction severity on the regional level, and psychological well-being in adolescents. Thus, the present study will provide novel 
information on how infection rates and infection control measures at the municipality level predict changes in psychosocial variables from before to during the pandemic.

\section{The COVID-19 pandemic in Norway}

Despite the relatively low COVID-19 related death rate in Norway, infection control measures in Norway have been similar to those in other European countries ${ }^{34}$ and include closure of schools, mandatory physical distancing, and severe restrictions on recreational activities. Also in Norway, working from home has been the rule since March 2020. Moreover, during the recent data collection for the present study in 2021 (January to March), COVID-19 infection rates in Norway were of the same magnitudes as in Portugal, Ireland, Iceland, Finland, Denmark, Germany, Belgium, and Italy ${ }^{34}$.

\section{The present study}

In summary, there is an urgent need for methodologically sound, large-scale population-based studies examining consequences of the long-lasting COVID-19 pandemic for adolescents. Using a unique, nationwide dataset comprising 227,258 adolescents, we examine the following three questions: What are the psychosocial consequences of the pandemic for adolescents, one year after its onset? Are adolescents from a disadvantaged background disproportionally affected by the pandemic? Do pandemic effects vary according to geographical variations in infection rates and restrictions? We expect that the pandemic has adverse effects on social relationships (friends, families), mental health, and outlook for the future. And in contrast, we expect to find a decrease in substance use and conduct problems. We expect screen time to have increased, whereas the effect of the pandemic on physical activity is unclear. Despite limited evidence for adolescents, we expect that the pandemic disproportionally affects girls, adolescents in poor families, and adolescents with low parental education. Finally, we expect the effects of the pandemic to vary with the infection rates and restriction measures on the municipality level.

\section{Results}

We used data from 227,258 adolescents from 157 municipalities in Norway, collected between 2014 and 2021. Table 1 presents information about the sample according to year of participation. In 2021, 86,597 adolescents participated. Because municipalities typically participate every third or fourth year in the data collection scheme, the number of municipalities included before 2021 was highest in 2018, 2017, and 2015. Response rates were high and ranged from $77 \%$ in 2021 to $85 \%$ in 2017, except of the year 2020. The response rate in that year was considerably lower (65\%) because some school classes did not succeed to participate before schools were closed on March 12 due to the pandemic.

Table 2 presents descriptive statistics for all outcome variables and sociodemographic characteristics across all data collection years. All psychosocial outcomes were scaled as percent of maximum possible (POMP) scores, which can be interpreted as the percentage of the maximum possible scores achievable on the scale ${ }^{35}$. On average, adolescents were highly satisfied with their social relationships, as they indicated satisfaction above $80 \%$ of the maximum score possible. They scored on average on the lower end of the scale for indicators of mental health, substance use, and conduct problems. Moreover, adolescents scored relatively high on physical activity and daily screen time (67\%), and $70 \%$ of the adolescents reported expecting a happy life in the future. Concerning socioeconomic status, $19 \%$ of the adolescents indicated that neither of their parents had higher education, and 5\% perceived their family's economic status to be poor. 
In a first set of analyses, we examined the effect of the pandemic for each outcome variable by using multilevel regression analyses to construct societal growth curves for all municipalities. Moreover, to estimate the effect of the pandemic, we included a dummy variable indicating participation in 2021, expressing the deviation of the outcome variable during the pandemic over and above the general trend as represented by the societal growth curves. Parental education, gender, and age were included as covariates. We did not include perceived family poverty as a covariate, because parts of the potential adverse effects of the pandemic may be due to increasing financial difficulties in some families. Table 3 presents growth parameters of the societal growth curves and the estimated effect of the pandemic; Fig. 1 presents the results graphically and shows rather stable levels of satisfaction with peer and parental relationships across the years from 2014 to 2021 . Moreover, analyses revealed no significant effect of the pandemic in 2021 on either peer or parental relationships, as the dummy variable assessing the average deviation in 2021 from the general trend line was not significant for peer or parental relationships. Depressive symptoms increased substantially from 2014 to 2018 and levelled out thereafter. Depressive symptoms were 2.13 POMP scores higher than expected in 2021, thereby indicating a significant adverse effect of the pandemic on depressive symptoms (see Table 3 and Fig. 1). Loneliness increased linearly from 2014 and thereafter, and increased as such also under the pandemic in 2021. However, no additional adverse effect of the pandemic on loneliness was observed. Concerning substance use, smoking behaviour did not change significantly as a result of the pandemic. In contrast, adolescents reported significantly decreased levels of alcohol intoxication and less use of cannabis in 2021, relative to what would have been expected according to the trend line (a decrease of 2.58 and 0.87 POMP scores, respectively). Physical activity decreased slightly through all years, and we did not see an effect of the pandemic. However, the pandemic had an effect on screen time, as with 1.69 POMP scores in 2021 it increased over and above an already increasing general time trend. Finally, adolescents were less optimistic about their own future during the pandemic, compared with what would be expected according to the general time trend (a decrease of 2.36 POMP scores).

Next, we examined whether adolescents from a disadvantaged background were disproportionally affected by the pandemic. For this purpose, we tested whether the pandemic effect dummy variable indicating changes over and above the general time trend was moderated by low parental education and perceived poverty. Fig. 2 shows the results of the interaction analyses from multilevel models by graphically presenting point estimates of pandemic effects according to sociodemographic characteristics. The results show that adolescents with low parental education were more affected in several domains: The pandemic had greater adverse effects on peer and parental relationships, depressive symptoms, and loneliness, as satisfaction with these relationships decreased more and depressive symptoms and loneliness increased more. There were no significant moderation effects of parental education on drug use and conduct problems; however, both physical activity and screen time decreased more among those with low parental education. Also, the decline in optimism about their future was greater in adolescents with low parental education. Similar results were found when examining perceived family poverty, with more severe adverse effects in the domains of social relationships and mental health for those who perceived the family's economic situation to be difficult. Additionally, adolescents who perceived their family to be poor showed a comparatively smaller decrease in smoking, cannabis use, and conduct problems. As was found for perceived family poverty, physical activity declined more with low parental education, whereas no differences between adolescents in poor families and other adolescents were found for screen time and optimism about their future.

When examining moderator effects for gender and age, we found girls and younger adolescents to be disproportionally affected by the pandemic as compared to boys and older adolescents (Fig. 2). Peer relationships and parental relationships decreased more for girls than boys, whereas depressive symptoms increased more. Moreover, smoking, alcohol intoxication, and conduct problems decreased less for girls relative to boys. Further, physical activity increased less, and screen time increased more for girls, compared to boys. Only loneliness showed an opposite trend, with boys 
more affected than girls by the pandemic. Finally, for all variables but cannabis use, older age was related to less adverse effects of the pandemic.

In a final set of multilevel analyses, we examined whether infection rates at the municipality level and the number of weeks with strict local restrictions moderated the effect of the pandemic by including cross-level interaction with these two municipality level variables in the model. Results showed no interaction effects for infection rates $(p>.05)$, indicating that municipality level infection rates were not related to changes from before to during the pandemic for any of the assessed psychosocial variables. When we examined restrictions, we found a significant interaction effect only for smoking, indicating that for each week with additional restrictions in schools in a municipality, smoking behavior decreased with 0.15 POMP scores more during the pandemic than in municipalities without such restrictions $(p<.01)$.

\section{Discussion}

By using a nationwide sample of 227,258 adolescents with measures before and one year into the COVID-19 pandemic, this study provides unique data on the potential effects of the pandemic on key psychosocial aspects in adolescents' lives. By using multilevel societal growth curves to adjust for general time trends, we show that depressive symptoms and screen time increased. Moreover, alcohol intoxication and cannabis use decreased, and adolescents were less optimistic about their future. Most strikingly, we see a consistent association between low parental education/perceived family poverty and adverse effects of the pandemic in several domains of adolescents' lives. In addition, girls and younger adolescents were more affected by the pandemic than boys and older adolescents. Finally, COVID-19 infection rates on the municipality level were not related to how adolescents were affected by the pandemic. Stricter restrictions on the municipality level were related only to a greater reduction in smoking and were not related to the other ten measures of psychosocial well-being assessed in this study.

\section{The effect of the COVID-19 pandemic on adolescents}

The results reveal that peer and parental relationships did not change substantially as an effect of the pandemic. These results are in contrast to concerns that peer relations and relationships within the family may suffer during the pandemic ${ }^{10-12}$. Instead, the results support voices suggesting that the pandemic, in addition to negative effects on social relationships, may also lead to positive changes, including increased amount of time and attention spent within the family ${ }^{13}$. The combination of these negative and positive changes may lead to no substantial overall changes in satisfaction with peer and parental relationships during the pandemic.

In the domain of mental health, the present study shows that the pandemic seems to have a negative effect on depressive symptoms. The results may be explained by the adverse effects of social isolation, the economic recession, and disruptions in mental health care services due to the pandemic ${ }^{1,2}$. The results are in line with research on adults showing substantial increases in mental health problems among adults early in the pandemic ${ }^{14-18}$. However, the increase of about 2.13 units on a scale from 0 to 100 shows that the effect is of a moderate magnitude and seems to be considerably smaller than what has been found in studies on adults. The rather moderate increases are in accordance with findings from longitudinal studies on adolescents from the beginning of the pandemic that observed rather small changes in mental health problems $s^{3,6}$. Interestingly, contrary to expectations, we found no adverse effect of the pandemic on adolescents' loneliness. This is possibly because adolescents' relationships with peers and parents did not deteriorate during the pandemic. This finding is also in line with a longitudinal study among Norwegian adults that observed stable or even falling loneliness trends during the pandemic ${ }^{36}$. 
From a public health perspective, the pandemic may have affected substance use in a positive way, indicated by the decline in adolescent alcohol intoxications and cannabis use during the pandemic. Physical distancing measures probably reduced the frequency of occasions where adolescents would have used drugs, such as parties and unsupervised meetings with other adolescents. The pandemic may also have forced adolescents to stay at home under parental supervision more frequently, thereby reducing the opportunities to use drugs. Research on adolescents' drug use during the pandemic is scarce; however, our results are in accordance with longitudinal research on adults in the $\mathrm{UK}^{20}$.

Physical activity did not change significantly as a result of the pandemic, whereas screen time increased. It may seem surprising that physical activity did not decrease, because adolescents' opportunities to participate in organized sports activities were severely restricted during the pandemic. However, we may see similar mechanisms as have been found in children and adolescents in Germany, where decreasing organized sports activities were counterbalanced by a substantial increase in recreational physical activities ${ }^{7}$. This potential mechanism is in accordance with evidence from Norway showing that recreational use of urban green space increased substantially during the pandemic ${ }^{37}$. Increased screen time outside school is in accordance with other studies measuring the effect of the pandemic on adolescents 7,23 and may be explained by more leisure time spent online and more frequent use of digital media to socialize in times of restricted opportunities for organized leisure time activities and physical distancing measures.

Finally, this is the first study to show that adolescents are now less optimistic about their own future than before the pandemic. We suggest that the pandemic may have challenged adolescents' feelings concerning physical safety and future economic security, which in turn may have increased worries about the future and decreased optimism.

In sum, the findings show negative effects of the pandemic on adolescents' mental health and expectations about their future, but the pandemic has also led to decreased substance use. The observed effect sizes seem to be rather small, with typical increases and decreases of few percentage points.

\section{Disproportional effect of the pandemic on disadvantaged adolescents}

The results show that the pandemic has hit disadvantaged adolescents the hardest. Adolescents with low parental education and those from poor families were more negatively affected in several domains, including peer and parental relationships, mental health, and physical activity. Poverty was additionally related to a smaller decrease in smoking, cannabis use, and conduct problems. Our results are in line with research showing that the poor and underprivileged suffer the most in times of crisis $^{28}$. Other studies have demonstrated that the less educated and the poor are economically more severely affected by the COVID-19 pandemic ${ }^{29,30}$. Building on these findings, our findings show that adolescents from families with low education and poor economic backgrounds are also affected in a variety of other life domains. The results clearly indicate the need for societal means and measures to reduce the negative impact of the pandemic for underprivileged groups.

Moreover, the results indicate that sociodemographic factors such as gender and age are additional sources of disparities in how the pandemic has affected adolescents' lives. The disproportional adverse effects on mental health for girls are in line with the notion that adolescent girls are more reactive and more likely to become depressed as a consequence of significant stress exposure than boys ${ }^{38}$. Our results are also in line with two studies demonstrating widening gender disparities for mental health during the pandemic in adolescents and adults ${ }^{4,14}$. We extend the literature by showing that the pandemic affects girls more severely than boys in other psychosocial domains as well, thereby indicating that gender disparities in the impact of the pandemic extend beyond the mental health domain. 
This study is among the first to find that younger adolescents are more affected by the pandemic than older adolescents. These results might be partially explained by the multiple changes associated with puberty that younger adolescents must have undergone; after all, puberty is characterized by dramatic changes in hormone levels that alter adolescents' sensitivity to exogenous stressors ${ }^{39}$ and as such, younger adolescents might be more sensitive to the negative impact of the pandemic. In contrast, older adolescents might have completed the majority of pubertal changes and acquired increased self-regulatory capacity ${ }^{33}$, which may make them less vulnerable to the effects of the pandemic than younger adolescents. It seems reasonable that as young people mature over the course of adolescence (i.e. acquire more cognitive, social, and emotional capacities), they may become better equipped to deal with the impact of the pandemic on psychosocial aspects of their lives.

\section{Few municipality level interaction effects}

The results indicated no effect of municipality level variations in infection rates, and also the extent of the imposed restriction measures was only related to smoking behaviour. We believe that such effects were largely not observed because infection rates varied only moderately in Norway. Moreover, by far the most restriction measures in Norway were implemented on the national level, and local variations may therefore have been of minor importance for adolescents' psychosocial well-being. An important future focus of research may be to examine the effects of infection rates and restrictions measures when comparing areas with larger variations in such figures, such as examining crosscountry differences.

\section{Strengths and limitations}

The study's strengths include the very large sample of adolescents and the repeated cross-sectional design with data collections before and during the pandemic using identical procedures and measures. Moreover, the comprehensive assessment of psychosocial factors in different life domains and the stringent adjustment for overall time trends are additional strengths. However, the results have to be interpreted in light of several limitations. First, concerning measurement issues, we assessed some of the variables (e.g. peer and parental relationships) with one-item instruments only. Future studies should include more comprehensive measures. We operationalized family poverty by an item on perceived family economic situation, and a more objective measure of family income would have been preferable. Moreover, the study did not conduct more extensive assessments of disadvantage, such as ethnic minority status or non-heterosexual orientations. Also, we did not directly assess age but only based on school grade. However, previous Norwegian studies found nearly perfect correlations between age and school grade ${ }^{40}$. Second, compared to other years of data collection, response rates were considerably lower in 2020, because some school classes did not succeed to participate before the lockdown. However, the risk of bias due to the lower response rate in 2020 is low because non-participation was primarily due to random factors such as when the survey was planned to be conducted. Third, generalizability of the results to other countries may be limited, because Norway has had comparably fewer COVID-19 related deaths than many other countries. However, restriction measures and infection rates have been similar to those in other European countries. Fourth, even though our analyses provide sound knowledge about changes in a variety of psychosocial variables about one year after the onset of the pandemic while accounting for general time trends, the study does not provide definite evidence of causal effects of the pandemic. We acknowledge that deviations from the general trend during the pandemic in 2021 may partly be caused by societal changes in this year that are unrelated to the COVID-19 pandemic.

\section{Conclusion}

This study provides solid evidence that as a result of the pandemic, adolescents' depressive symptoms and time spent in front of a screen increased, whereas adolescents' optimism about their future decreased. Alcohol intoxication and 
cannabis use decreased. The effects were of only moderate size and may in themselves not indicate alarming adverse effects of the pandemic. However, of concern is the consistent finding that girls, young adolescents, and adolescents with a low socioeconomic background seemed to be affected more by the pandemic. This finding indicates that the pandemic, in line with other crises, disproportionally affects the disadvantaged. And similar to other crises, the disproportionally negative effects may be long-lasting and affect the disadvantaged negatively far beyond the duration of the pandemic ${ }^{24}$. It is therefore imperative to develop and implement measures such as economic support and public health interventions that are aimed at buffering the effects of the pandemic for adolescents and their families with low socioeconomic resources. Moreover, the particular vulnerability of girls and the youngest adolescents have to be taken into account when developing interventions. Norway is a typical social democratic welfare state ${ }^{41}$, characterized by rather extensive social welfare services and benefits, including a universal health insurance system, which may buffer the economic, health, and psychosocial effects of the pandemic on the disadvantaged. Arguably, the COVID-19 pandemic may therefore lead to even larger social disparities among adolescents in other countries that have less generous social security systems. Research on the effect of the pandemic on social disparities in other countries will therefore be an important research focus in the future.

\section{Methods}

\section{Data and participants}

The present study used data from Norwegian nationwide Ungdata surveys. Ungdata is a national data collection scheme designed to conduct youth surveys at the national and municipal levels in Norway. It is regarded as the most wide-ranging source of data on adolescent health and well-being in Norway, and adolescents in almost all municipalities are regularly assessed, typically every third year. The Ungdata data collection scheme was started in 2010 but has been fully implemented for all junior and senior high school students (grades 8 to 13 , students aged 13 to 18) since 2014. Participating students were invited to complete an electronic questionnaire in class, covering various aspects of young people's lives, including social relationships, mental health, substance use, health behaviour, normbreaking behaviour, exposure to negative life events, and leisure activities.

Data collection was conducted each spring. Also in 2020, data collection started in January but was discontinued when schools were closed in Norway on March 12. At that time, only some of the participating municipalities had finished considerable parts of the data collection. In 2021, 204 municipalities participated in Ungdata from January to the end of March. Of those, data from 43 municipalities were not used because one or several of outcomes, predictors, or controls were not assessed in these municipalities. Furthermore, four municipalities that had not conducted any Ungdata surveys before 2021 were excluded. Of the remaining 157 municipalities, 43, 70, 41, and three municipalities had conducted one, two, three, and four data collections before 2021, respectively. In total, we used data from $N=$ 227,258 adolescents in 157 municipalities who had participated in Ungdata in 2021 and at least at one previous data collection. All participants and their parents were informed that participation in Ungdata is voluntary. Parents had the possibility to reserve their children from participation. All relevant ethical regulations were followed, and the study was recommended by the Department of Psychology's internal research ethics committee at the University of Oslo (reference \# 13710027).

\section{Measures}

Social relationships. Peer relationships were assessed by one item asking how satisfied the respondents were with their friendships with peers. Parental relationship was assessed in a similar way by asking how satisfied the respondents were with their parents. Both items were measured by a 5-point scale ranging from very unsatisfied to very satisfied. 
Mental health. Depressive symptoms were measured by Kandel and Davies' 6-item Depressive Mood Inventory ${ }^{42}$. This measure was derived from the widely used Hopkins Symptom Checklist ${ }^{43}$ and assesses depressive symptoms during the preceding week on a 4-point scale from affected not at all to affected extremely. In the present study internal consistency was $a=.89$. Loneliness was assessed by one item on feelings of loneliness in the last week, with the same response options.

Substance use and health behaviour. Adolescents' smoking behaviour was assessed, which we categorized into those who did not smoke (1), smoked less than once a week (2), smoked every week but not daily (3), and daily smokers (4). Alcohol intoxication was assessed by asking how often over the past year participants had consumed so much alcohol that they clearly felt intoxicated. We also assessed cannabis use in the past year. Conduct problems were assessed by a 5-item instrument on the frequency of stealing, vandalism, tagging, truancy, and not paying at public transportation or events. A composite score of the five items was computed. Response options for alcohol intoxication, cannabis use, and conduct problems items were on a 5-point scale ranging from neverto more than 10 times.

Physical activity and screen time. Respondents' physical activity was assessed by asking how often they engaged in physical activity that caused them to breathe harder or break a sweat, on a scale ranging from 1 (never) to 6 (at least five times a week). Screen time was assessed by asking respondents about their daily use of screens outside of school with response options ranging from 1 (no time) to 6 (more than 3 hours).

Future life expectations. Future life expectations were assessed by one item about whether respondents expected to live a good and happy life. Response options were yes, no, and don't know. We contrasted those who responded that they expected to live a good and happy life (yes) with all other adolescents (no and don't know).

Indicators of socioeconomic status and other demographics. Low parental education was operationalized by whether at least one of the parents had a university or college education or not. Perceived family poverty was measured by asking "Has your family's economic situation been good or bad during the past two years?", with five response options ranging from always good to always bad. We contrasted those who perceived the family's economic situation as mostly bad or always bad with all other adolescents. Gender was assessed. For anonymity concerns, only school grade (Grades 8 to 13) but not age was assessed. In the Norwegian school system, attendance in school grades is strictly organized by birth cohorts, and staying back (repeating a grade) due to poor academic performance is generally not practiced. Therefore, we used school grade as an indicator of age, where Grade 8 corresponds to age 13 and Grade 13 corresponds to age 18 .

Municipality level variables. Municipality level data on total COVID-19 infection rates per 100,000 residents from the onset of the pandemic to March 31, 2021 were obtained from the Norwegian Surveillance System for Communicable Diseases. Because restrictions to control the spread of the COVID-19 pandemic varied across municipalities, we used a database of all restrictions on the municipality level that is operated by one of the largest national newspapers in Norway, Verdens Gang. The database is continuously updated by direct contact with the municipalities and by monitoring municipality webpages, official documents, and official announcements. We identified all registered restrictions that were directed towards junior and senior high schools in the municipalities, as they were the only restrictions that were specifically directed towards adolescents, and we calculated the number of days with stricter restrictions in municipalities than what had been imposed by national authorities.

\section{Analyses}

To provide a scaling of all psychosocial outcome variables that is easily interpretable and allows comparison across measurements, we transformed all dependent variables into POMP scores ${ }^{35}$. Thus, in line with the POMP score 
approach, variables were rescaled with minimum and maximum possible scores of 0 and 100 , respectively. Scores can be interpreted as the percentage of the maximum possible score achievable on the scale $\mathrm{e}^{35}$.

Due to the hierarchical structure of the data, with individuals nested within municipalities observed repeatedly over time, we used multilevel regression models in all analyses. In particular, we applied the 'societal growth curve' approach to multilevel modelling, as introduced by Fairbrother ${ }^{8}$. This approach was specifically developed for designs such as Ungdata, where multiple geographical units (e.g. municipalities) are observed across time, but at each point of observation, a different representative cross-sectional sample of individuals is drawn from the population ${ }^{44,45}$. The method thus allows assessment of how aggregated individual characteristics develop over time within repeatedly sampled higher-level units (i.e. municipalities). More specifically, using multilevel linear regression analyses, we constructed growth curves for each municipality to model time trends on the municipality level from 2014 to 2021 for indicators of psychosocial well-being. All models were estimated as random-intercept multilevel linear regressions with individuals at the lowest level, clustered within municipality years at the middle level, and municipalities at the highest level. The inclusion of random intercepts at the municipality-years level and municipality level was also supported empirically, since variability in the random intercepts at the higher levels was found to be significantly different from zero for all outcomes $(p<.05)$.

The overall pattern of change over time was modelled as a curvilinear trend, through inclusion of both linear and quadratic terms for number of years that had passed since the first included survey wave in 2014. Additionally, we measured the effect of the pandemic in 2021 over and above the curvilinear development by including a dummy variable for the 2021 wave (coded 1 for participating in the 2021 data wave and 0 for participation in all other data waves; for another application of this approach, see ${ }^{44}$ ). Due to convergence issues, societal growth curve slope parameters and the pandemic effects were fixed to be the same across all municipalities. More specifically, the societal growth model was specified by means of the following equation

$Y_{i t j}=\beta_{0}+\beta_{7} t i m e_{t j}+\beta_{2} t i m e_{t j}^{2}+\beta_{3} d u m m y_{t j}+v_{0 j}+u_{0 t j}+\varepsilon e_{i t j}$

with $\quad e_{i t j} \sim N\left(0, s_{e}^{2}\right)$

$u_{0 t j} \sim N\left(0, s_{u}^{2}\right)$

$v_{0 j} \sim N\left(0, s_{v}^{2}\right)$

where $Y_{i t j}$ represents a psychosocial characteristic for adolescent $i$ at data collection wave $t$ in municipality $j$. $\beta_{0}$ represents the grand intercept across all municipalities, and $\beta_{1}$ and $\beta_{2}$ represent the linear slope and quadratic slope of the societal growth curve, respectively. $\beta_{3}$ is the coefficient for the dummy, indicating the deviation of the dependent variable in the pandemic year of 2021 over and above the general trend as expressed by the growth curve. Moreover, the model includes random intercepts for the municipality $\left(v_{0 j}\right)$ and municipality-year level $\left(u_{0 t j}\right)$. The two, together with the individual-level error term $\left(e_{i t}\right)$, are assumed to be distributed normally, with a mean of 0 .

Because all dependent variables were recoded into POMP scores, the pandemic effect can be interpreted in terms of percentage-point change of the percentage of the maximum possible score achievable on the scale ${ }^{35}$. We controlled for parental education, gender, and age (not shown in the equation) to adjust for individual-level compositional differences that may have affected the societal growth curves or the estimated effect of the pandemic ${ }^{46}$.

To examine whether the pandemic disproportionally affected particular groups of adolescents, we included interaction terms of the pandemic effect variable with the individual-level predictors of parental educational background, perceived 
family poverty, gender, and age. We then calculated point estimates of these conditional effects for specific values of moderator variables ${ }^{47}$. We also explored whether the impact of the pandemic varied with municipality infection rates and extent of imposed restrictions. For this purpose, we included cross-level interactions of the pandemic effect with infection rates and extent of restriction measures.

In line with best practices for multilevel modelling ${ }^{48}$, all predictors and controls were grand-mean centred to facilitate interpretation of the estimates. We used $\mathrm{R}$ version 4.0.3 for all analyses. All multilevel regressions were conducted using the Ime4 package for $\mathrm{R}$, version $1.1 .26^{49}$. The interplot package for $\mathrm{R}$, version $0.2 .3^{50}$ was used to estimate conditional effects for interaction analyses.

\section{Declarations}

\section{Data availability}

The data that support the findings of this study are available from Norwegian Social Research (NOVA) but restrictions apply to the availability of these data, which were used under license for the current study, and so are not publicly available. Data are however available from the authors upon reasonable request and with permission of Norwegian Social Research (NOVA).

\section{Code availability}

The code for all analyses reported in the manuscript is available on request.

\section{References}

1 Golberstein, E., Wen, H. \& Miller, B. F. Coronavirus Disease 2019 (COVID-19) and mental health for children and adolescents. JAMA Pediatr. 174, 819-820 (2020).

2 Holmes, E. A. et al. Multidisciplinary research priorities for the COVID-19 pandemic: a call for action for mental health science. Lancet Psychiatr. 7, 547-560 (2020).

3 Barendse, M. E. A. et al. Longitudinal change in adolescent depression and anxiety symptoms from before to during the COVID-19 pandemic: An international collaborative of 12 samples. PsyArXiv, doi:

10.31234/osf.io/hn31237us (2021).

4 Magson, N. R. et al. Risk and protective factors for prospective changes in adolescent mental health during the COVID-19 pandemic. J. Youth Adolesc. 50, 44-57 (2021).

5 Dumas, T. M., Ellis, W. \& Litt, D. M. What does adolescent substance use look like during the COVID-19 pandemic? Examining changes in frequency, social contexts, and pandemic-related predictors. J. Adolesc. Health 67, 354-361 (2020).

6 Hafstad, G. S., Sætren, S. S., Wentzel-Larsen, T. \& Augusti, E.-M. Adolescents' symptoms of anxiety and depression before and during the Covid-19 outbreak. A prospective population-based study of teenagers in Norway. Lancet Reg. Health Eur. 5, doi: 10.1016/j.lanepe.2021.100093 (2021).

7 Schmidt, S. C. E. et al. Physical activity and screen time of children and adolescents before and during the COVID-19 lockdown in Germany: a natural experiment. Sci. Rep. 10, 21780 (2020). 
8 Fairbrother, M. Two multilevel modeling techniques for analyzing comparative longitudinal survey datasets. Polit. Sci. Res. Meth. 2, 119-140 (2014).

9 Orben, A., Tomova, L. \& Blakemore, S.-J. The effects of social deprivation on adolescent development and mental health. Lancet Child. Adolesc. Health 4, 634-640 (2020).

10 Prime, H., Wade, M. \& Browne, D. T. Risk and resilience in family well-being during the COVID-19 pandemic. Am. Psychol. 75, 631-643 (2020).

11 Cluver, L. et al. Parenting in a time of COVID-19. Lancet 395, e64 (2020).

12 Lee, S. J. \& Ward, K. P. Stress and parenting during the coronavirus pandemic. (Parenting in Context Research Lab, University of Michigan, Michigan, 2020).

13 Bruining, H., Bartels, M., Polderman, T. J. C. \& Popma, A. COVID-19 and child and adolescent psychiatry: an unexpected blessing for part of our population? Eur. Child Adolesc. Psychiatr., doi: 10.1007/s00787-00020-0157800785 (2020).

14 Pierce, M. et al. Mental health before and during the COVID-19 pandemic: a longitudinal probability sample survey of the UK population. Lancet Psychiatr. 7, 883-892 (2020).

15 Twenge, J. M. \& Joiner, T. E. Mental distress among U.S. adults during the COVID-19 pandemic. J. Clin. Psychol. 76, 2170-2182 (2020).

16 McGinty, E. E., Presskreischer, R., Han, H. \& Barry, C. L. Psychological distress and loneliness reported by US adults in 2018 and april 2020. JAMA 324, 93-94 (2020).

17 Ebrahimi, O. V., Hoffart, A. \& Johnson, S. U. Physical distancing and mental health during the COVID-19 pandemic: Factors associated with psychological symptoms and adherence to pandemic mitigation strategies. Clin. Psychol. Sci., doi: 10.1177/2167702621994545 (2021).

18 Czeisler, M. É., Howard, M. E. \& Rajaratnam, S. M. W. Mental health during the COVID-19 pandemic: challenges, populations at risk, implications, and opportunities. Am. J. Health Promot. 35, 301-311 (2021).

19 Rehm, J. et al. Alcohol use in times of the COVID 19: Implications for monitoring and policy. Drug Alcohol Rev. 39, 301-304 (2020).

20 Bann, D. et al. Changes in the behavioural determinants of health during the coronavirus (COVID-19) pandemic: gender, socioeconomic and ethnic inequalities in 5 British cohort studies. medRxiv, doi: 10.1101/2020.1107.1129.20164244 (2020).

21 Boman, J. H. \& Gallupe, O. Has COVID-19 changed crime? Crime rates in the united states during the pandemic. Am. J. Crim. Justice 45, 537-545 (2020).

22 Hall, G., Laddu, D. R., Phillips, S. A., Lavie, C. J. \& Arena, R. A tale of two pandemics: How will COVID-19 and global trends in physical inactivity and sedentary behavior affect one another? Prog. Cardiovasc. Dis. 64, 108-110 (2021).

23 Xiang, M., Zhang, Z. \& Kuwahara, K. Impact of COVID-19 pandemic on children and adolescents' lifestyle behavior larger than expected. Prog. Cardiovasc. Dis. 63, 531-532 (2020). 
24 Arthi, V. \& Parman, J. Disease, downturns, and wellbeing: Economic history and the long-run impacts of COVID-

19. Explor. Econ. Hist. 79, doi: 10.1016/j.eeh.2020.101381 (2021).

25 Mackenbach, J. P. et al. Socioeconomic inequalities in health in 22 European countries. N. Engl. J. Med. 358, 2468-2481 (2008).

26 Chetty, R. et al. The association between income and life expectancy in the United States, 2001-2014. JAMA 315, 1750-1766 (2016).

27 Viner, R. M. et al. Adolescence and the social determinants of health. Lancet 379, 1641-1652 (2012).

28 Heggeb $\varnothing$, K., Tøge, A. G., Dahl, E. \& Berg, J. E. Socioeconomic inequalities in health during the Great Recession: A scoping review of the research literature. Scand. J. Publ. Health 47, 635-654 (2018).

29 Adams-Prassl, A., Boneva, T., Golin, M. \& Rauh, C. Inequality in the impact of the coronavirus shock: Evidence from real time surveys. J. Publ. Econ. 189, 104245 (2020).

30 Perry, B. L., Aronson, B. \& Pescosolido, B. A. Pandemic precarity: COVID-19 is exposing and exacerbating inequalities in the American heartland. PNAS 118, e2020685118 (2021).

31 Benner, A. D. \& Mistry, R. S. Child development during the COVID-19 pandemic through a life course theory lens. Child Dev. Perspect. 14, 236-243 (2020).

32 Crick, N. R. \& Zahn-Waxler, C. The development of psychopathology in females and males: Current progress and future challenges. Dev. Psychopathol. 15, 719-742 (2003).

33 Steinberg, L. Cognitive and affective development in adolescence. Trends Cognit. Sci. 9, 69-74 (2005).

34 World Health Organization. WHO coronavirus (COVID-19) dashboard, <https://covid19.who.int/> (2021).

35 Cohen, P., Cohen, J., Aiken, L. S. \& West, S. G. The problem of units and the circumstance for POMP. Multivar Behav Res 34, 315-346 (1999).

36 Hansen, T. et al. Locked and lonely? A longitudinal assessment of loneliness before and during the COVID-19 pandemic in Norway. Scand. J. Publ. Health, doi: 10.1177/1403494821993711 (2021).

37 Venter, Z. S., Barton, D. N., Gundersen, V., Figari, H. \& Nowell, M. Urban nature in a time of crisis: recreational use of green space increases during the COVID-19 outbreak in Oslo, Norway. Environ. Res. Lett. 15, 104075 (2020).

38 Nolen-Hoeksema, S. Emotion regulation and psychopathology: The role of gender. Annu. Rev. Clin. Psychol. 8, 161-187 (2012).

39 Blakemore, S.-J., Burnett, S. \& Dahl, R. E. The role of puberty in the developing adolescent brain. Hum. Brain Mapp. 31, 926-933 (2010).

40 Aarø, L. E. et al. Mental and somatic complaints among adolescents. J. Norweg. Med. Assoc. 121, 2923-2927 (2001).

41 Esping-Andersen, G. The three worlds of welfare capitalism. (Princeton University Press, 1990). 
42 Kandel, D. B. \& Davies, M. Epidemiology of depressive mood in adolescents. Arch. Gen. Psychiatr. 39, 1205-1212 (1982).

43 Derogatis, L. R., Lipman, R. S., Rickels, K., Uhlenhuth, E. H. \& Covi, L. The Hopkins Symptom Checklist (HSCL): A self-report symptom inventory. Behav. Sci. 19, 1-15 (1974).

44 Meuleman, B., Davidov, E. \& Billiet, J. Modeling multiple-country repeated cross-sections. A societal growth curve model for studying the effect of the economic crisis on perceived ethnic threat. Meth. Data Anal. 12, 185-210 (2018).

45 Schmidt-Catran, A. W. \& Fairbrother, M. The random effects in multilevel models: Getting them wrong and getting them right. Eur. Sociol. Rev. 32, 23-38 (2016).

46 Snijders, T. \& Bosker, R. Multilevel analysis. An introduction to basic and advanced mutilevel modeling. 2nd edn, (Sage, 2012).

47 Brambor, T., Clark, W. R. \& Golder, M. Understanding interaction models: Improving empirical analyses. Polit. Anal. 14, 63-82 (2006).

48 Enders, C. K. \& Tofighi, D. Centering predictor variables in cross-sectional multilevel models: A new look at an old issue. Psychol. Methods 12, 121-138 (2007).

49 Bates, D., Mächler, M., Bolker, B. \& Walker, S. Fitting linear mixed-effects models using Ime4. J. Stat. Software 67 (2015).

50 Solt, F. \& Hu, Y. Interplot: Plot the effects of variables in interaction terms, <https://cran.rproject.org/web/packages/interplot/vignettes/interplot-vignette.html> (2021).

\section{Tables}

Table 1

Sample characteristics according to data collection year

\begin{tabular}{lccccccccc}
\hline & 2014 & 2015 & 2016 & 2017 & 2018 & 2019 & 2020 & 2021 & Total \\
\hline $\begin{array}{l}\text { Number of individual } \\
\text { observations }\end{array}$ & 11,719 & 24,694 & 10,555 & 44,103 & 30,246 & 8,792 & 10,552 & 86,597 & 227,258 \\
$\begin{array}{l}\text { Number of participating } \\
\text { municipalities }\end{array}$ & 31 & 61 & 33 & 75 & 66 & 16 & 36 & 157 & 157 \\
$\begin{array}{l}\text { Average response rate } \\
(\%)\end{array}$ & 80 & 81 & 78 & 85 & 81 & 81 & 65 & 77 & 79 \\
\hline
\end{tabular}

Note. Data from 2020 were collected before the lockdown due to the COVID-19 outbreak (i.e. before March 12, 2020).

Table 2

Descriptive statistics and intercorrelations for variables under study 


$M \quad S D$ Range (1) (2) $(3)$ (4) (5)

Social

relationships

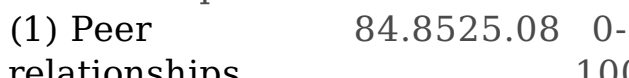

(2) Parental $\quad 85.6626 .93 \quad 0-\quad 0.55$

relationship 100

Mental health

(3) Depressive

symptoms

$35.8026 .650-\quad-0.23-0.23$

(4) Loneliness $\quad 28.3433 .34 \quad 0-\quad-0.31-0.210 .68$

Substance use and conduct problems
(5) Smoking
$5.0916 .52 \quad 0-\quad-0.03-0.110 .130 .08$
100
(6) Alcohol
$21.0532 .95 \quad 0-\quad 0.01-0.070 .180 .080 .41$
intoxication
100
(7) Cannabis use
$3.3814 .89 \quad 0-\quad-0.04-0.100 .120 .080 .440 .35$
100
(8) Conduct
problems
$8.2513 .04 \quad 0-\quad-0.07-0.180 .240 .160 .380 .400 .39$

Physical activity

and screen time

(9) Physical activity71.5424.27 0-

$0.100 .11-0.14-0.15-0.09-0.04-0.05-0.05$

100

(10) Screen time $67.0223 .04 \quad 0-\quad-0.05-0.070 .210 .170 .070 .090 .070 .16-0.16$

Future

expectations

(11) Expecting a 70.4045.65 0- 0.18 0.19-0.42-0.37-0.07-0.04-0.07-0.13 0.17-0.15

happy future 100

Sociodemographics

(12) \% with low $\quad 19 \% \quad$-0.04-0.06 $0.040 .050 .080 .080 .030 .04-0.130 .03-0.05$

parental education

(13) \% with $\quad 5 \% \quad-0.10-0.160 .170 .150 .080 .050 .070 .11-0.080 .05-0.130 .12$

perceived family

poverty

(14) \% girls

$50 \%$

-0.04-0.04 0.32 0.21-0.06 0.02-0.07-0.07-0.11-0.04-0.07-0.010.03

(15) Age

$15.301 .6013-$

$0.00-0.020 .150 .080 .180 .530 .150 .16-0.080 .07-0.040 .130 .050 .03$

Note. Intercorrelations of $r=|.01|$ or above are statistically significantly different from zero at $p<.001$. Continuous measures (except for age) are scaled as percent of maximum possible (POMP) scores, with a minimum score of 0 and a maximum achievable score of 100. $M=$ mean; $S D=$ standard deviation.

Table 3

Societal growth curve estimates from 2014 to 2021 and estimated effects of the pandemic for 11 indicators of psychosocial well-being 


\begin{tabular}{|c|c|c|c|c|c|}
\hline & \multicolumn{3}{|c|}{$\begin{array}{c}\text { Societal growth curve estimates of time } \\
\text { trends }\end{array}$} & \multicolumn{2}{|c|}{$\begin{array}{l}\text { Estimated effects of the } \\
\text { pandemic }\end{array}$} \\
\hline & $\begin{array}{c}\text { Intercept } \\
\left(\beta_{0}\right)\end{array}$ & $\begin{array}{l}\text { Linear slope } \\
\qquad\left(\beta_{1}\right)\end{array}$ & $\begin{array}{l}\text { Quadratic slope } \\
\qquad\left(\beta_{2}\right)\end{array}$ & $\beta_{3}$ & $\begin{array}{l}\text { 95\% confidence } \\
\text { interval }\end{array}$ \\
\hline \multicolumn{6}{|l|}{ Social relationships } \\
\hline Peer relationships & $84.83 * * *$ & -0.16 & 0.02 & -0.55 & {$[-1.45 ; 0.35]$} \\
\hline Parental relationships & $85.88 * * *$ & $0.31^{* *}$ & 0.01 & -0.48 & {$[-1.40 ; 0.45]$} \\
\hline \multicolumn{6}{|l|}{ Mental health } \\
\hline Depressive symptoms & $36.08 * * *$ & $0.38 * *$ & $-0.21 * * *$ & $2.13^{* * *}$ & {$[0.99 ; 3.27]$} \\
\hline Loneliness & $27.93 * * *$ & $0.77 * * *$ & -0.02 & 0.70 & {$[-0.62 ; 2.02]$} \\
\hline \multicolumn{6}{|l|}{$\begin{array}{l}\text { Drug use and conduct } \\
\text { problems }\end{array}$} \\
\hline Smoking & $4.94 * * *$ & 0.03 & 0.04 & -0.42 & {$[-1.26 ; 0.43]$} \\
\hline Alcohol intoxication & $20.42 * * *$ & $0.64^{* *}$ & $0.26 * * *$ & $-2.58 * *$ & {$[-4.41 ;-0.74]$} \\
\hline Cannabis use & $2.78 * * *$ & $0.26 * * *$ & 0.03 & $-0.87 * *$ & {$[-1.52 ;-0.22]$} \\
\hline Conduct problems & $7.29 * * *$ & $0.24 * *$ & 0.04 & 0.61 & {$[-0.09 ; 1.30]$} \\
\hline \multicolumn{6}{|l|}{$\begin{array}{l}\text { Physical activity and } \\
\text { screen time }\end{array}$} \\
\hline Physical activity & $71.22 * * *$ & $-0.26 *$ & -0.01 & 0.57 & {$[-0.61 ; 1.75]$} \\
\hline Screen time & $66.47 * * *$ & $1.70 * * *$ & $0.07 *$ & $1.69 * *$ & {$[0.65 ; 2.73]$} \\
\hline \multicolumn{6}{|l|}{ Future expectations } \\
\hline $\begin{array}{l}\text { Expecting a happy } \\
\text { future }\end{array}$ & $69.25^{* * *}$ & -0.18 & $0.26^{* * *}$ & $-2.36 * *$ & {$[-4.12 ;-0.60]$} \\
\hline
\end{tabular}

Note. ${ }^{*} p<.05,{ }^{* *} p<.01 * * * p<.001$. Due to mean centering, the intercept of societal growth curves $\left(\beta_{0}\right)$ can be interpreted as the estimated value between 2018 and 2019 (2018.5). Linear and quadratic slope parameters represent change from one year to the next.

\section{Figures}



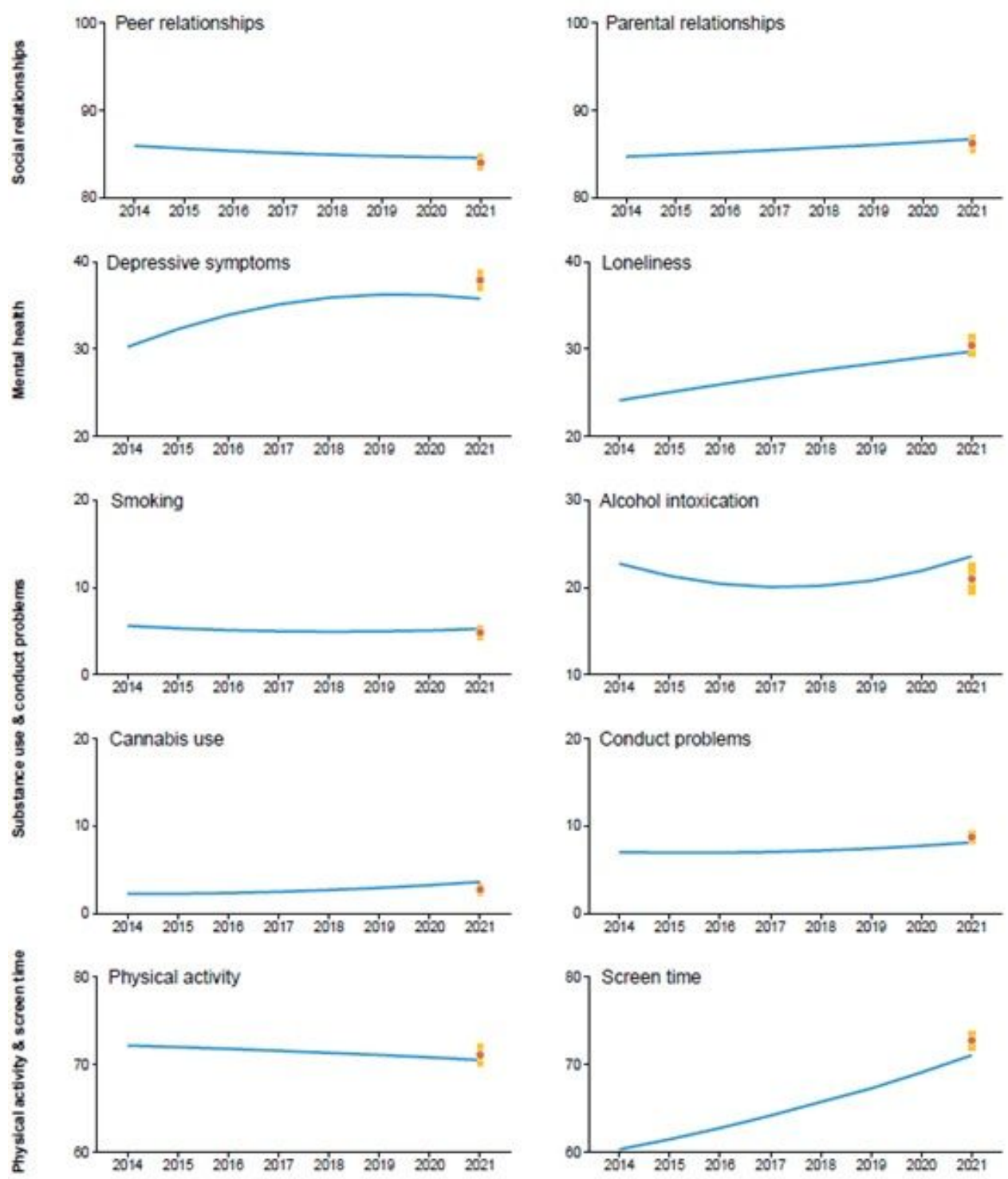

$\frac{\frac{2}{2}}{\frac{5}{5}}$

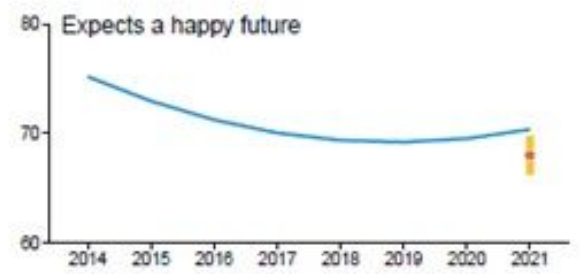

Figure 1

Time trends in psychosocial aspects of adolescents' lives from 2014 to 2021 and the estimated effect of the pandemic. Note. The blue line indicates the time trends in adolescent psychosocial well-being from 2014 to 2021 as estimated by societal growth curves. The red dot represents the average estimated value during the pandemic in 2021 , and the $95 \%$ confidence interval is represented by orange bars. Data from 2020 were collected before the lockdown due to the COVID-19 outbreak (i.e. before March 12, 2020). 

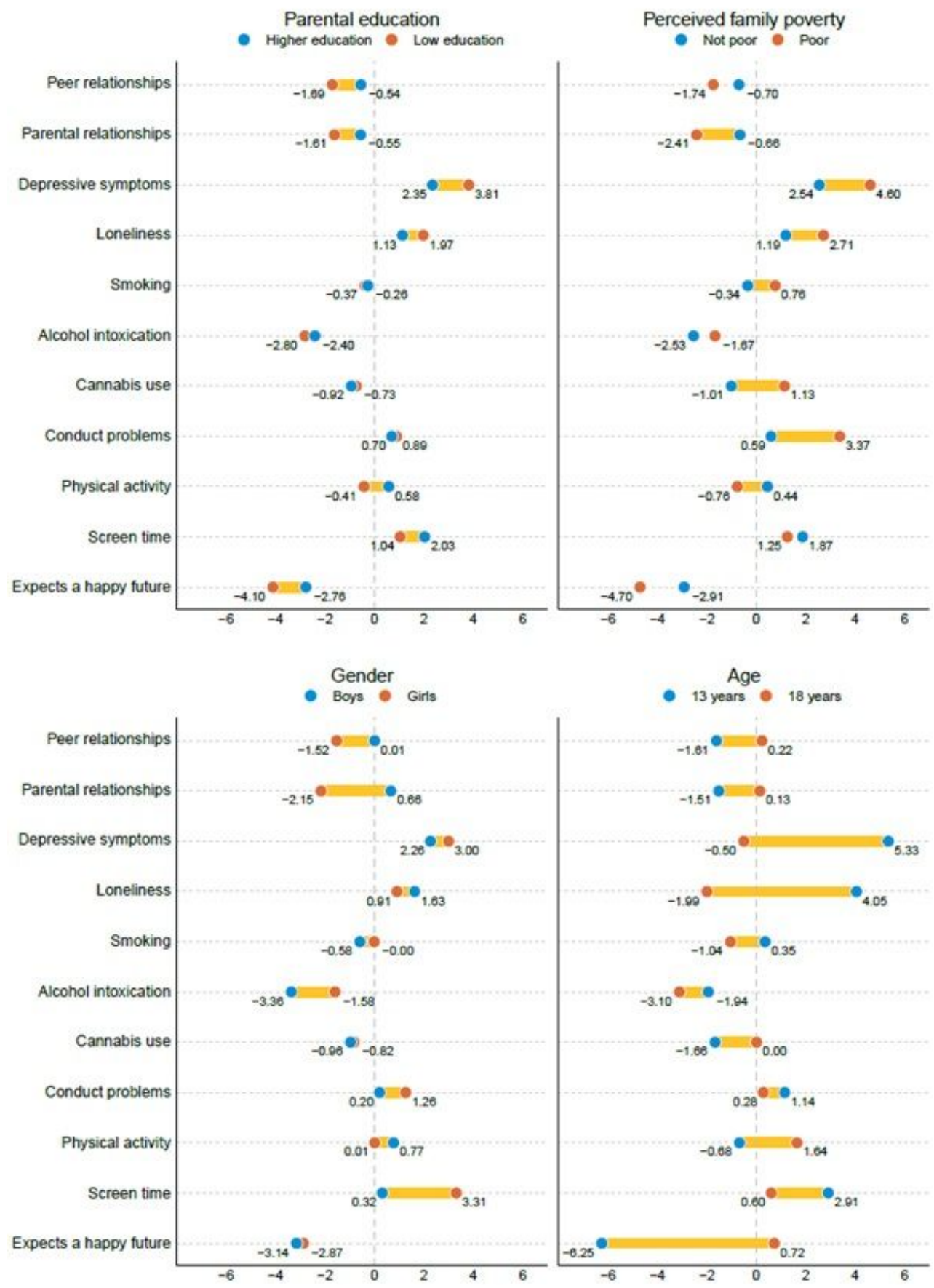

Figure 2

The effect of the pandemic according to indicators of disadvantage, gender, and age. Note. Blue and red dots indicate point estimates of the effect of the pandemic for specific groups of adolescents, as estimated by conditional effects analysis. The orange bars represent statistically significant differences $(p<.05)$ of the pandemic effects for different groups of adolescents. Tests of significance were provided by interaction analyses in multilevel models.

\section{Supplementary Files}

This is a list of supplementary files associated with this preprint. Click to download.

- reportingsummary.pdf 